\title{
Control of Continuous -Time LTI Systems by Means of Structurally Constrained Controllers
}

\author{
Javad Lavaei and Amir G. Aghdam \\ Department of Electrical and Computer Engineering, Concordia University \\ Montréal, QC Canada H3G 1 M8 \\ \{j_lavaei,aghdam\}@ece.concordia.ca
}

\begin{abstract}
This paper deals with the characterization of the fixed modes of multi-channel systems with respect to linear time-invariant (LTI) structurally constrained controllers. Fixed modes can be found numerically for any LTI system with respect to any given control structure, using a random number generator. The existing analytical methods, however, are not capable of characterizing the fixed modes in the most general case of non-strictly proper systems with non-block diagonal (i.e., overlapping) control structure efficiently. The notion of decentralized overlapping fixed mode (DOFM) is introduced in this paper to address the above problem in the most general case. To this end, the knowledge of the overlapping control structure is translated into a bipartite graph, whose vertices correspond to the input and output vectors of various control channels. An efficient technique is extracted from the obtained graph to identify the DOFMs of the system. It is to be noted that a system is stabilizable via an appropriate LTI decentralized overlapping controller if and only if it does not have any unstable DOFM. Moreover, it is shown how those modes which are not DOFMs can be placed freely in the complex plane using a proper LTI decentralized overlapping controller. The efficacy of this work is demonstrated through an example.
\end{abstract}

\section{Introduction}

In control of large-scale systems, it is often desired to have some form of decentralization. In fact, for such systems it is not realistic to assume that all output measurements can be employed to construct every input. Problems of this kind appear, for example, in electric power systems, communication networks, large space structures, robotic systems, economic systems and traffic networks, to name only a few (Li and Wang, 2005; Movsichoff, Lagoa, and Che, 2005; Lavaei, Momeni, and Aghdam, 2007; Becerril and Aghdam, 2007).

In the past three decades, the problem of decentralized control has been thoroughly investigated in the literature, and a variety of its aspects are studied (Wang and Davison, 1973; Davison and Chang, 1990; Pichai, Sezer, and Siljak, 1984; Gong and Aldeen, 1997). More recently, the problem of decentralized overlapping control has attracted several researchers (Zecevic and Siljak , 2005; Bakule, Rodellar, and Rossell, 2003). This problem has applications in many real-world interconnected systems such as chemical processes, power systems, and traffic

\footnotetext{
1 This work has been supported by the Natural Sciences and Engineering Research Council of Canada under grant RGPIN-262127-03.
}

networks (Li et al., 1999). The decentralized overlapping control is fundamentally used in two types of problems:

i) when the subsystems of an interconnected system (referred to as overlapping subsystems) share some states (Iftar, 1993, 1991; Siljak and Zecevic, 2005). In this case, it is usually desired that the structure of the controller matches the overlapping structure of the system (Siljak and Zecevic, 2005);

ii) when there are some limitations on the availability of certain outputs to generate the control signal for any specific input in the sense that such a data transmission is very costly or sometimes impossible. This case frequently occurs in practice; for instance, in the formation of spacecraft in deep space where communication between different spacecraft is crucially exorbitant, and where some information may not be available for certain spacecraft due to the shadow phenomenon (Smith and Hadaegh, 2002).

The control constraint in both types of problems can be represented by a binary information flow matrix. In the case when this matrix is block diagonal with the entries of the main diagonal blocks all equal to 1 , the control structure is indeed the conventional decentralized configuration (this control structure will be simply referred to as decentralized control throughout this paper). One 
particular structural constraint for the controller which is investigated intensively in the literature, corresponds to an information flow matrix with the entries of the main diagonal blocks, as well as the last block column and the last block row all equal to 1 . This configuration is often referred to as bordered block-diagonal structure (BBD) or block array structure (BAS), and has found several practical applications (Siljak and Zecevic, 2005; Groumpos, 1994; Leros and Groumpos, 1987).

Designing a decentralized overlapping controller for an interconnected system has been investigated extensively in the literature by transforming the system into an expanded form whose corresponding control structure is decentralized (Siljak and Zecevic, 2005; Zecevic and Siljak , 2005; Stankovic, Stanojevic, Siljak , 2000). This expanded system should be obtained in such a way that its subsystems be connected to each other by means of weak interconnections. In this case, the interconnections between the subsystems are ignored first, and the local controllers for the disjoint subsystems are designed accordingly. As the last step of the design procedure, the decentralized controller obtained should be contracted in order to take the desirable overlapping form for the original system. The mathematical framework for the expansion and contraction is often referred to as the Inclusion Principle (Chu and Siljak, 2005).

It is known that the stability of the expanded decentralized closed-loop system is not equivalent to that of the original overlapping closed-loop system. Thus, the expanded system might not preserve the structural properties of the original system. More precisely, when the original system is stabilizable by means of a static (dynamic) LTI decentralized overlapping controller, the expanded system will likely not be stabilizable via static (dynamic) LTI decentralized controller, unless it is devised meticulously. It is shown in Bakule, Rodellar, and Rossell (2001) that if the original system and its subsystems are all controllable and observable, the expanded system can be obtained in such a way that it is controllable and observable. Moreover, it is asserted in Chu and Siljak (2005) that the properties of controllability, stabilizability, observability, detectability, and stability of the invariant zeros can be transferred from the original system to the expanded one, if a proper transformation is exploited.

If the original system has some unstable fixed modes with respect to the LTI decentralized overlapping controllers, the available control design methods using Inclusion Principle are inefficient. Hence, the characterization of such undesirable modes is of particular importance. It is notable that this characterization can primarily facilitate the decentralized overlapping control design by using the expansion-contraction methods, and can potentially solve some of the open problems in this area (see Siljak and Zecevic (2005) for one of these open problems regarding the contraction conditions).
The notion of decentralized fixed mode (DFM) was introduced in Wang and Davison (1973) to identify the modes of a strictly proper system which are fixed with respect to any LTI decentralized controller. This notion was later extended to general proper systems in Davison and Chang (1990). Several methods are proposed in the literature to characterize the DFMs of any strictly proper system (Davison and Wang, 1985; Anderson, 1982; Anderson and Clements, 1981). It is shown in Pichai, Sezer, and Siljak (1984) that the modes of a strictly proper system which are fixed with respect to any LTI decentralized overlapping controller can be obtained from the DFMs of another system with some redundant outputs. However, this method has two main drawbacks. First, it has not tackled the problem for general proper systems. Moreover, since it duplicates solely the outputs of the system (not also the inputs), it often results in a new decentralized system of unnecessarily large dimension.

It is well known that graph theory provides a powerful tool in handling diverse problems, including various problems in control systems. It is shown in Reinschke (1988) how a square matrix can be characterized using a graph-theoretic approach, and also how its determinant can be found accordingly. Similar techniques are used therein to model any LTI control system in terms of a graph which is utilized to obtain its transfer function and other properties. Different aspects of the control design such as pole-placement, disturbance rejection, structural controllability and structurally fixed modes, are investigated in the literature in the framework of graph theory (Reinschke, 1988; Siljak, 1991; Mayeda, 1981; Lafferriere, Caughman, and Williams, 2004).

The present work is concerned with the characterization of the fixed modes of general proper systems with respect to any class of LTI decentralized overlapping controllers. To this end, the notion of decentralized overlapping fixed mode (DOFM) is defined to characterize such modes systematically. This is carried out via a graph-theoretic approach which is utilized to translate the knowledge of the desired control structure into several equivalent bipartite graphs. The DOFMs of the system can then be obtained from any of these formed graphs. Thus, the designer can choose the graph whose corresponding computational complexity is the least in order to find such modes.

This paper is organized as follows. The structurally constrained control problem is formulated in Section 2. The information of the desired structure of the controller is translated into some graphs in Section 3, and certain transformation matrices are then obtained. These matrices are employed in Section 4 to characterize the DOFMs of a general proper system efficiently. A necessary and sufficient condition for the stabilizability of the system by means of structurally constrained LTI controllers is also presented in Section 4 followed by a pole placement 
technique. An illustrative example is given in Section 5 to clarify the results of the present work. Finally, some concluding remarks are provided in Section 6 .

\section{Problem formulation}

Consider an LTI $\nu$-channel system $\mathcal{S}$ with the following state-space representation:

$$
\begin{aligned}
\dot{x}(t) & =A x(t)+\sum_{i=1}^{\nu} B_{i} u_{i}(t) \\
y_{i}(t) & =C_{i} x(t)+\sum_{j=1}^{\nu} D_{i j} u_{j}(t), \quad i \in \bar{\nu}:=\{1,2, \ldots, \nu\}
\end{aligned}
$$

where $x(t) \in \Re^{n}$ is the state, and $u_{i}(t) \in \Re^{m_{i}}$ and $y_{i}(t) \in$ $\Re^{r_{i}}, i \in \bar{\nu}$, are the input and the output of its $i^{\text {th }}$ channel, respectively. Define the following matrices:

$$
\begin{aligned}
B & :=\left[\begin{array}{llll}
B_{1} & B_{2} & \cdots & B_{\nu}
\end{array}\right], \\
C & :=\left[\begin{array}{cccc}
C_{1}^{T} & C_{2}^{T} & \cdots & C_{\nu}^{T}
\end{array}\right]^{T}, \\
D & :=\left[\begin{array}{ccc}
D_{11} & \cdots & D_{1 \nu} \\
\vdots & \ddots & \vdots \\
D_{\nu 1} & \cdots & D_{\nu \nu}
\end{array}\right]
\end{aligned}
$$

Define also:

$$
m:=\sum_{i=1}^{\nu} m_{i}, \quad r:=\sum_{i=1}^{\nu} r_{i}
$$

It is desired to stabilize the system $\mathcal{S}$ via a structurally constrained controller. These constraints determine which outputs $y_{j}(t)(j \in \bar{\nu})$ are available to construct any specific input $u_{i}(t)(i \in \bar{\nu})$ of the system. In order to simplify the formulation of the control constraint, a matrix $\mathcal{K}$ with binary elements is defined, where its $(i, j)$ block entry, $i, j \in \bar{\nu}$, is a $m_{i} \times r_{j}$ matrix whose elements are all equal to 1 if the output $y_{j}(t)$ can contribute to the construction of the input $u_{i}(t)$, and is a $m_{i} \times r_{j}$ zero matrix otherwise. The matrix $\mathcal{K}$ represents the control constraint, and will be referred to as the information flow matrix.

To specify the structural constraint of the control for the system, the corresponding information flow matrix will be enclosed within parentheses as an argument throughout the paper, if necessary. For instance, $\mathcal{S}(\mathcal{K})$ indicates that the structure of the controller to be designed for the system $\mathcal{S}$ is to comply with the information flow matrix $\mathcal{K}$. In the special case, when the entries of the matrix $\mathcal{K}$ are all equal to 1 , the corresponding controller is centralized, and when $\mathcal{K}$ is block diagonal, the corresponding controller is decentralized.
Characterizing those modes of the system $\mathcal{S}$ which are fixed with respect to any LTI controller complying with the control structure $\mathcal{K}$ will be addressed in the sequel using an efficient technique.

\section{Computing the transformation matrices}

Define the control interaction structure $\mathbf{K}$ as a matrix whose $(i, j)$ block entry, $i, j \in \bar{\nu}$, is a $m_{i} \times r_{j}$ matrix denoted by $k_{i j}$ if the output $y_{j}(t)$ can contribute to the construction of the input $u_{i}(t)$, and is a $m_{i} \times r_{j}$ zero matrix otherwise. Note that $k_{i j}$ represents a component of the controller, which transforms the output $y_{j}(t)$ to the input $u_{i}(t)$. Note also that not only does the interaction structure matrix $\mathbf{K}$ convey the information of the matrix $\mathcal{K}$, it also labels the control components. The matrices $\mathcal{K}$ and $\mathbf{K}$ will henceforth be used for different purposes.

Procedure 1 Construct the graph $\mathcal{G}$ as follows:

(1) Define two sets of $\nu$ vertices. Label the sets as set 1 and set 2 , and the vertices in each set as vertex 1 to vertex $\nu$.

(2) For any $i, j \in \bar{\nu}$, connect the $i^{\text {th }}$ vertex of set 1 to the $j^{\text {th }}$ vertex of set 2 with an edge if the $(i, j)$ block entry of $\mathcal{K}$ is not a zero matrix. Label this edge with $k_{i j}$.

Procedure 2 Partition the graph $\mathcal{G}$ into a set of complete bipartite subgraphs such that each edge of the graph $\mathcal{G}$ appears in only one of the subgraphs. It is to be noted that this partition may require some of the vertices of the graph $\mathcal{G}$ to appear in multiple subgraphs.

It can be easily verified that Procedure 2 does not necessarily lead to a unique graph. Denote all the graphs which can be obtained through this procedure with $\mathcal{G}_{1}, \mathcal{G}_{2}, \ldots, \mathcal{G}_{l}$.

The following procedure constructs a matrix $\mathbf{K}_{\mu}$ corresponding to the graph $\mathcal{G}_{\mu}$, for any $\mu \in \bar{l}:=\{1,2, \ldots, l\}$.

Procedure 3 Label the complete bipartite subgraphs of $\mathcal{G}_{\mu}(\mu \in \bar{l})$ as subgraphs 1 to $\nu_{\mu}$. Consider subgraph number $\sigma\left(\forall \sigma \in\left\{1,2, \ldots, \nu_{\mu}\right\}\right)$. Label those vertices of this subgraph which belong to set 1 as vertex $1, \ldots, \eta_{\sigma}^{\mu}$. This group of vertices will be referred to as subset 1 (corresponding to subgraph number $\sigma$ ). Similarly, label those vertices which belong to set 2 of this subgraph as vertex $1, \ldots, \bar{\eta}_{\sigma}^{\mu}$, and define subset 2 accordingly. Define $\mathbf{K}_{\mu}$ as a block diagonal matrix, where its $(\sigma, \sigma)$ block entry, $\sigma=1, \ldots, \nu_{\mu}$, is a matrix itself, whose $(i, j)$ block entry is equal to the gain of the edge connecting vertex $i$ of subset 1 to vertex $j$ of subset 2 in subgraph number $\sigma$ of $\mathcal{G}_{\mu}$, for any $i \in\left\{1, \ldots, \eta_{\sigma}^{\mu}\right\}$ and $j \in\left\{1, \ldots, \bar{\eta}_{\sigma}^{\mu}\right\}$. Denote the dimension of the $(\sigma, \sigma)$ block entry of $\mathbf{K}_{\mu}$ with $m_{\sigma}^{\mu} \times r_{\sigma}^{\mu}$, for $\sigma=1,2, \ldots, \nu_{\mu}$, and the dimension of $\mathbf{K}_{\mu}$ with $m^{\mu} \times r^{\mu}$. 
As an example, consider a two-channel system with the following control interaction structure:

$$
\mathbf{K}=\left[\begin{array}{cc}
k_{11} & 0 \\
k_{21} & k_{22}
\end{array}\right]
$$

The graph $\mathcal{G}$ corresponding to the matrix $\mathbf{K}$ (introduced in Procedure 1) is depicted in Figure 1.

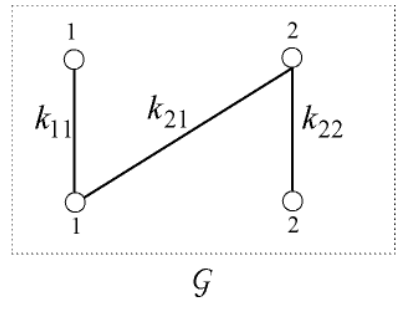

Fig. 1. The graph $\mathcal{G}$ of the system $\mathcal{S}$ with the matrix $\mathbf{K}$ given by (4).

The graphs $\mathcal{G}_{1}, \mathcal{G}_{2}$ and $\mathcal{G}_{3}$ obtained from Procedure 2 are sketched in Figure 2. As it can be seen from this figure, some of the vertices are recurring in each graph.
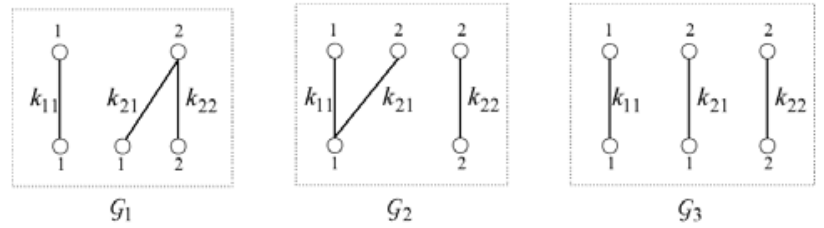

Fig. 2. The graphs $\mathcal{G}_{1}, \mathcal{G}_{2}$ and $\mathcal{G}_{3}$ corresponding to the system $\mathcal{S}$ with the matrix $\mathbf{K}$ given by (4).

Therefore, the matrices $\mathbf{K}_{1}, \mathbf{K}_{2}$ and $\mathbf{K}_{3}$ can be attained using Procedure 3 as follows:

$$
\begin{aligned}
& \mathbf{K}_{1}=\left[\begin{array}{ccc}
k_{11} & 0 & 0 \\
0 & k_{21} & k_{22}
\end{array}\right], \mathbf{K}_{2}=\left[\begin{array}{cc}
k_{11} & 0 \\
k_{21} & 0 \\
0 & k_{22}
\end{array}\right], \\
& \mathbf{K}_{3}=\left[\begin{array}{ccc}
k_{11} & 0 & 0 \\
0 & k_{21} & 0 \\
0 & 0 & k_{22}
\end{array}\right]
\end{aligned}
$$

Remark 1 It can be easily concluded from Procedures 1, 2 and 3 that there exists an onto mapping between the nonzero block entries of the matrix $\mathbf{K}_{\mu}, \mu \in \bar{l}$, and those of the matrix $\mathbf{K}$.

Theorem 1 There exist constant binary matrices $\Phi_{\mu}$ and $\bar{\Phi}_{\mu}$ satisfying the following relation:

$$
\mathbf{K}=\Phi_{\mu} \mathbf{K}_{\mu} \bar{\Phi}_{\mu}
$$

for any $\mu \in \bar{l}$.

Sketch of proof: It is straightforward to show (by using Procedures 1, 2 and 3) that the matrix $\mathbf{K}_{\mu}$ can alternatively be constructed from $\mathbf{K}$ through a sequence of matrices $\mathbf{K}_{1}^{\mu}, \mathbf{K}_{2}^{\mu}, \ldots, \mathbf{K}_{L-1}^{\mu}, \mathbf{K}_{L}^{\mu}$, where $\mathbf{K}=\mathbf{K}_{1}^{\mu}, \mathbf{K}_{\mu}=$ $\mathbf{K}_{L}^{\mu}$, and moreover $\mathbf{K}_{j+1}^{\mu}$ is obtained from $\mathbf{K}_{j}^{\mu}$ by one of the following two operations (for any $j \in\{1,2, \ldots, L-$ $1\})$ :

1. Swapping either two columns or two rows of the $\operatorname{matrix} \mathbf{K}_{j}^{\mu}$.

2. Splitting one of the rows (or columns) of $\mathbf{K}_{j}^{\mu}$ denoted by $v$, into two row vectors $v_{1}$ and $v_{2}$, i.e., $v=\left[\begin{array}{ll}v_{1} & v_{2}\end{array}\right]\left(\right.$ or $\left.v=\left[\begin{array}{ll}v_{1} & v_{2}\end{array}\right]^{\prime}\right)$. Then, replacing that row (or column) with $\left[\begin{array}{ll}v_{1} & 0\end{array}\right]$ (or $\left.\left[\begin{array}{ll}v_{1} & 0\end{array}\right]^{\prime}\right)$, where 0 represents a zero row vector, and inserting another row (or column) equal to $v=\left[\begin{array}{ll}0 & v_{2}\end{array}\right]$ (or $\left.v=\left[\begin{array}{ll}0 & v_{2}\end{array}\right]^{\prime}\right)$ into the matrix.

It can be easily shown that for any $j \in\{1, \ldots, L-1\}$, there exist matrices $\Phi_{j}^{\mu}$ and $\bar{\Phi}_{j}^{\mu}$ such that $\mathbf{K}_{j}^{\mu}=\Phi_{j}^{\mu} \mathbf{K}_{j+1}^{\mu} \bar{\Phi}_{j}^{\mu}$. The matrices $\Phi_{\mu}$ and $\bar{\Phi}_{\mu}$ can now be obtained from the following equations:

$$
\Phi_{\mu}=\Phi_{1}^{\mu} \Phi_{2}^{\mu} \cdots \Phi_{(L-1)}^{\mu}, \quad \bar{\Phi}_{\mu}=\bar{\Phi}_{(L-1)}^{\mu} \bar{\Phi}_{(L-2)}^{\mu} \cdots \bar{\Phi}_{1}^{\mu}
$$

This completes the proof.

Theorem 1 states that there exist matrices $\Phi_{\mu}$ and $\bar{\Phi}_{\mu}$ such that they satisfy the equation (6). However, the procedure proposed in the proof of the theorem for obtaining $\Phi_{\mu}$ and $\bar{\Phi}_{\mu}$ is computationally inefficient. The following theorem presents a more efficient approach to obtain $\Phi_{\mu}$ and $\bar{\Phi}_{\mu}$.

Theorem 2 Choose at least one nonzero block entry from each block column and each block row of $\mathbf{K}_{\mu}, \mu \in \bar{l}$, and denote them with $k_{i_{1} j_{1}}, k_{i_{2} j_{2}}, \ldots, k_{i_{p} j_{p}}$. Suppose that $k_{i_{q} j_{q}}, q=1,2, \ldots, p$, is the $\left(i_{q}^{\prime}, j_{q}^{\prime}\right)$ block entry of the matrix $\mathbf{K}_{\mu}$. The relations:

$$
\Pi_{i_{q}^{\prime}}=\left[\begin{array}{c}
0_{m_{1} \times m_{i_{q}}} \\
0_{m_{2} \times m_{i_{q}}} \\
\vdots \\
0_{m_{\left(i_{q}-1\right)} \times m_{i_{q}}} \\
I_{m_{i_{q}}} \\
0_{m_{\left(i_{q}+1\right)} \times m_{i_{q}}} \\
\vdots \\
0_{m_{\nu} \times m_{i_{q}}}
\end{array}\right], \quad \bar{\Pi}_{j_{q}^{\prime}}=\left[\begin{array}{c}
0_{r_{j_{q}} \times r_{1}} \\
0_{r_{j_{q}} \times r_{2}} \\
\vdots \\
0_{r_{j_{q}} \times r_{\left(j_{q}-1\right)}} \\
I_{r_{j_{q}}} \\
0_{r_{j_{q}} \times r_{\left(j_{q}+1\right)}} \\
\vdots \\
0_{r_{j_{q}} \times r_{\nu}}
\end{array}\right]^{T}
$$

hold for any $q \in\{1,2, \ldots, p\}$, where $\Pi_{i_{q}^{\prime}}$ and $\bar{\Pi}_{j_{q}^{\prime}}$ denote 
the $i_{q}^{\text {th }}$ block column of $\Phi_{\mu}$ and the $j_{q}^{\text {th }}$ block row of $\bar{\Phi}_{\mu}$, respectively.

Proof: It is shown in Theorem 1 that the matrices $\Phi_{\mu}$ and $\bar{\Phi}_{\mu}$ exist to satisfy the equation (6). As a result, this equation holds for any arbitrary values for the block entries $k_{\sigma_{1} \sigma_{2}}, \sigma_{1}, \sigma_{2} \in \bar{\nu}$. Replace all block entries $k_{\sigma_{1} \sigma_{2}}$ 's in the equation (6), except $k_{i_{q} j_{q}}$, with zero matrices. It can be concluded from (6) that:

$$
\tilde{\mathbf{K}}_{i_{q} j_{q}}=\Pi_{i_{q}^{\prime}} k_{i_{q} j_{q}} \bar{\Pi}_{j_{q}^{\prime}}
$$

where $\tilde{\mathbf{K}}_{i_{q} j_{q}}$ is obtained from $\mathbf{K}$ by replacing all of its block entries with zero matrices, except for its $\left(i_{q}, j_{q}\right)$ block entry $k_{i_{q} j_{q}}$. The proof follows immediately from the equation $(9)$.

\section{Linear time-invariant control law}

In this section, it is desired to find conditions for the existence of a structurally constrained stabilizing LTI controller for the system $\mathcal{S}(\mathcal{K})$. Furthermore, a procedure is given to achieve pole placement using a proper LTI control law complying with $\mathcal{K}$.

Definition 1 Define $\mathcal{S}_{\mu}, \mu \in \bar{l}$, as a system with the following state-space representation:

$$
\begin{aligned}
& \dot{\mathbf{x}}_{\mu}(t)=A \mathbf{x}_{\mu}(t)+\mathbf{B}^{\mu} \mathbf{u}_{\mu}(t) \\
& \mathbf{y}_{\mu}(t)=\mathbf{C}^{\mu} \mathbf{x}_{\mu}(t)+\mathbf{D}^{\mu} \mathbf{u}_{\mu}(t)
\end{aligned}
$$

where the parameters of the above system are related to those of the original system $\mathcal{S}$ through the following equations:

$$
\mathbf{B}^{\mu}=B \Phi_{\mu}, \quad \mathbf{C}^{\mu}=\bar{\Phi}_{\mu} C, \quad \mathbf{D}^{\mu}=\bar{\Phi}_{\mu} D \Phi_{\mu}
$$

$\mathbf{u}_{\mu}(t) \in \Re^{m^{\mu}}$ and $\mathbf{y}_{\mu}(t) \in \Re^{r^{\mu}}$ are the input and the output of $\mathcal{S}_{\mu}$, respectively, and $\mathbf{x}_{\mu}(0)=x(0)$. For any $\mu \in \bar{l}$, define the information flow matrix $\mathcal{K}_{\mu}$ for the system $\mathcal{S}_{\mu}$ as a matrix obtained from $\mathbf{K}_{\mu}$ by replacing its nonzero block entry $k_{i j}$, with a $m_{i} \times r_{j}$ matrix whose entries are all equal to one, for any $i, j \in \bar{\nu}$.

Definition 2 Consider two arbitrary systems $\mathcal{S}_{d_{1}}$ and $\mathcal{S}_{d_{2}}$ associated with the information flow matrices $\mathcal{K}_{d_{1}}$ and $\mathcal{K}_{d_{2}}$, where $\mathcal{S}_{d_{1}}$ and $\mathcal{S}_{d_{2}}$ are of the same order and have the same initial state. Let $\mathbf{M}$ denote a given set of controllers. The systems $\mathcal{S}_{d_{1}}\left(\mathcal{K}_{d_{1}}\right)$ and $\mathcal{S}_{d_{2}}\left(\mathcal{K}_{d_{2}}\right)$ are called analogous with respect to $\mathbf{M}$ if for any controller $K_{d_{1}}$ in $\mathbf{M}$ complying with the information flow matrix $\mathcal{K}_{d_{1}}$, there also exists a controller $K_{d_{2}}$ in $\mathbf{M}$ complying with the information flow matrix $\mathcal{K}_{d_{2}}$ (and vice versa) such that the state of the system $\mathcal{S}_{d_{1}}$ under the controller $K_{d_{1}}$ is equivalent to the state of $\mathcal{S}_{d_{2}}$ under $K_{d_{2}}$, for all $t \geq 0$.
Theorem 3 For any $\mu \in \bar{l}$, the systems $\mathcal{S}_{\mu}\left(\mathcal{K}_{\mu}\right)$ and $\mathcal{S}(\mathcal{K})$ are analogous with respect to the set of all LTI controllers.

Remark 2 The set of all LTI controllers in the above theorem refers to any LTI controller whose input-output description can be expressed via a transfer function. Obviously, this set encompasses any LTI control law in the form of state feedback, static and dynamic output feedback, and observer-based control.

Proof of Theorem 3: Denote the transfer function matrix of any nonzero control component $k_{i j}$ with $K_{i j}(s), i, j \in$ $\bar{\nu}$ (the dimension of $K_{i j}(s)$ is the same as $k_{i j}$ but the control function itself is yet to be designed). Replace the block $k_{i j}$ with $K_{i j}(s)$ in the matrices $\mathbf{K}$ and $\mathbf{K}_{\mu}$ for any $i, j \in \bar{\nu}$, and denote the resultant control transfer function matrices with $K(s)$ and $K_{\mu}(s)$, respectively. It can be easily concluded from Theorem 1 that:

$$
K(s)=\Phi_{\mu} K_{\mu}(s) \bar{\Phi}_{\mu}
$$

Assume the control transfer function matrix $K(s)$ is such that the matrix $I_{r}-D K(s)$ is nonsingular. It is evident that the state of the system $\mathcal{S}$ under the controller $K(s)$ satisfies the following equation (in the Laplace domain):

$$
X(s)=\left(s I_{n}-A-B K(s)\left(I_{r}-D K(s)\right)^{-1} C\right)^{-1} x(0)
$$

On the other hand, it can be easily verified that $I_{r^{\mu}}-\bar{\Phi}_{\mu} D \Phi_{\mu} K_{\mu}(s)$ is nonsingular due to the assumption $\operatorname{det}\left(I_{r}-D K(s)\right) \neq 0$. Similarly, the state of the system $\mathcal{S}_{\mu}$ under the controller $K_{\mu}(s)$ can be obtained as follows:

$$
\begin{aligned}
\mathbf{X}_{\mu}(s) & =\left(s I_{n}-A-\mathbf{B}^{\mu} K_{\mu}(s)\right. \\
& \left.\times\left(I_{r^{\mu}}-\mathbf{D}^{\mu} K_{\mu}(s)\right)^{-1} \mathbf{C}^{\mu}\right)^{-1} \mathbf{x}_{\mu}(0)
\end{aligned}
$$

Using the equations (11) and (12), one can write:

$$
\begin{aligned}
B K(s)\left(I_{r}-\right. & D K(s))^{-1} C=B \Phi_{\mu} K_{\mu}(s) \bar{\Phi}_{\mu} \\
& \times\left(I_{r}-D \Phi_{\mu} K_{\mu}(s) \bar{\Phi}_{\mu}\right)^{-1} C \\
& =B \Phi_{\mu} K_{\mu}(s)\left(I_{r^{\mu}}-\bar{\Phi}_{\mu} D \Phi_{\mu} K_{\mu}(s)\right)^{-1} \bar{\Phi}_{\mu} C \\
& =\mathbf{B}^{\mu} K_{\mu}(s)\left(I_{r^{\mu}}-\mathbf{D}^{\mu} K_{\mu}(s)\right)^{-1} \mathbf{C}^{\mu}
\end{aligned}
$$

The proof follows from the relations (13), (14), and (15).

Corollary 1 For any $\mu \in \bar{l}$, the systems $\mathcal{S}_{\mu}\left(\mathcal{K}_{\mu}\right)$ and $\mathcal{S}(\mathcal{K})$ are analogous with respect to the set of all static LTI controllers.

Proof: The proof is omitted due to its similarity to the proof of Theorem 3 (note that the set of static controllers 
is a subset of the general LTI controllers; thus, the result of Corollary 1 is not trivial).

Remark 3 It can be easily concluded from Theorem 3 and Corollary 1 that all of the systems $\mathcal{S}(\mathcal{K}), \mathcal{S}_{1}\left(\mathcal{K}_{1}\right), \mathcal{S}_{2}\left(\mathcal{K}_{2}\right), \ldots, \mathcal{S}_{l}\left(\mathcal{K}_{l}\right)$ are analogous with respect to the set of LTI controllers, as well as the set of static LTI controllers. As a result, in order to design a continuous-time dynamic (or static) LTI controller for the system $\mathcal{S}$ with respect to the information flow structure $\mathcal{K}$ to achieve any design objective (such as pole placement), one can equivalently design a continuoustime LTI controller for the system $\mathcal{S}_{\mu}, \mu \in \bar{l}$, with respect to the information flow structure $\mathcal{K}_{\mu}$, to attain the same objective. The mapping between the components of $\mathbf{K}$ and $\mathbf{K}_{\mu}$ (derived from the equation (6)) can then be used to find the corresponding controller for the system $\mathcal{S}(\mathcal{K})$. The important advantage of this indirect design procedure is that the information flow structure $\mathcal{K}_{\mu}$ is block diagonal, and hence the problem is converted to the conventional decentralized control design problem, which can be handled by the existing methods (Davison and Chang, 1990; Ravi, Rosenthal, and Wang, 1995).

Now, partition the matrices $\mathbf{B}^{\mu}, \mathbf{C}^{\mu}$ and $\mathbf{D}^{\mu}, \mu \in \bar{l}$, as follows:

$$
\begin{aligned}
& \mathbf{B}^{\mu}=\left[\begin{array}{llll}
\mathbf{B}_{1}^{\mu} & \mathbf{B}_{2}^{\mu} & \cdots & \mathbf{B}_{\nu_{\mu}}^{\mu}
\end{array}\right], \\
& \mathbf{C}^{\mu}=\left[\begin{array}{c}
\mathbf{C}_{1}^{\mu} \\
\mathbf{C}_{2}^{\mu} \\
\cdots \\
\mathbf{C}_{\nu_{\mu}}^{\mu}
\end{array}\right], \quad \mathbf{D}^{\mu}=\left[\begin{array}{ccc}
\mathbf{D}_{1,1}^{\mu} & \cdots & \mathbf{D}_{1, \nu_{\mu}}^{\mu} \\
\vdots & \ddots & \vdots \\
\mathbf{D}_{\nu_{\mu}, 1}^{\mu} & \cdots & \mathbf{D}_{\nu_{\mu}, \nu_{\mu}}^{\mu}
\end{array}\right]
\end{aligned}
$$

where:

$$
\mathbf{B}_{i}^{\mu} \in \Re^{m_{i}^{\mu}}, \quad \mathbf{C}_{i}^{\mu} \in \Re^{r_{i}^{\mu}}, \quad \mathbf{D}_{i j}^{\mu} \in \Re^{r_{i}^{\mu} \times m_{j}^{\mu}}
$$

for any $i, j \in\left\{1,2, \ldots, \nu_{\mu}\right\}$ (note that $m_{i}^{\mu}$ and $r_{i}^{\mu}$ are defined in Procedure 3 ).

Theorem 4 Consider an arbitrary region $\mathcal{R}$ in the complex plane. There exists an LTI decentralized overlapping controller for the system $\mathcal{S}(\mathcal{K})$ to place all modes of the resultant closed-loop system inside the region $\mathcal{R}$, except for those modes which are DFMs of the system $\mathcal{S}_{\mu}$ with respect to $\mathcal{K}_{\mu}, \mu \in \bar{l}$.

Proof: As pointed out in Remark 3, the systems $\mathcal{S}(\mathcal{K})$ and $\mathcal{S}_{\mu}\left(\mathcal{K}_{\mu}\right)$ are equivalent in terms of pole placement capabilities. On the other hand, it results from the definition of DFM (Wang and Davison, 1973) that all of the modes of the system $\mathcal{S}_{\mu}\left(\mathcal{K}_{\mu}\right)$ except for its DFMs can be placed arbitrarily by using a proper LTI controller. This completes the proof.
Definition 3 Define decentralized overlapping fixed modes $(D O F M)$ of $\mathcal{S}(\mathcal{K})$ as those modes of the system $\mathcal{S}$ which are fixed with respect to any LTI controller with the information flow structure $\mathcal{K}$.

Theorem 4 states that the DOFMs of $\mathcal{S}(\mathcal{K})$ and the DFMs of $\mathcal{S}_{\mu}\left(\mathcal{K}_{\mu}\right), \forall \mu \in \bar{l}$ are the same. Hence, the DOFMs of $\mathcal{S}(\mathcal{K})$ can be obtained from any of the systems $\mathcal{S}_{1}\left(\mathcal{K}_{1}\right), \ldots, \mathcal{S}_{l}\left(\mathcal{K}_{l}\right)$. The following procedure is used to determine the DOFMs of the system $\mathcal{S}(\mathcal{K})$ from the DFMs of the system $\mathcal{S}_{\mu}\left(\mathcal{K}_{\mu}\right), \mu \in \bar{l}$.

Procedure 4 Consider any arbitrary integer $g$ belonging to $\bar{l}$. Let $\operatorname{sp}(A)$ denote the set of eigenvalues of $A$. The mode $\lambda \in \operatorname{sp}(A)$ is a DOFM of the system $\mathcal{S}$ with respect to the information flow matrix $\mathcal{K}$, if there exists a permutation of $\left\{1,2, \ldots, \nu_{\mu}\right\}$ denoted by the distinct integers $i_{1}, i_{2}, \ldots, i_{\nu_{\mu}}$, such that the rank of the matrix:

$$
\left[\begin{array}{ccccc}
A-\lambda I_{n} & \mathbf{B}_{i_{1}}^{\mu} & \mathbf{B}_{i_{2}}^{\mu} & \ldots & \mathbf{B}_{i_{q}}^{\mu} \\
\mathbf{C}_{i_{q+1}}^{\mu} & \mathbf{D}_{i_{q+1}, i_{1}}^{\mu} & \mathbf{D}_{i_{q+1}, i_{2}}^{\mu} & \ldots & \mathbf{D}_{i_{q+1}, i_{q}}^{\mu} \\
\mathbf{C}_{i_{q+2}}^{\mu} & \mathbf{D}_{i_{q+2}, i_{1}}^{\mu} & \mathbf{D}_{i_{q+2}, i_{2}}^{\mu} & \ldots & \mathbf{D}_{i_{q+2}, i_{q}}^{\mu} \\
\vdots & \vdots & \vdots & \ddots & \vdots \\
\mathbf{C}_{i_{\nu_{\mu}}}^{\mu} & \mathbf{D}_{i_{\nu_{\mu}}, i_{1}}^{\mu} & \mathbf{D}_{i_{\nu_{\mu}}, i_{2}}^{\mu} & \ldots & \mathbf{D}_{i_{\nu_{\mu}}, i_{q}}^{\mu}
\end{array}\right]
$$

is less than $n$ for some $\mu \in\left\{0,1, \ldots, \nu_{\mu}\right\}$.

Remark 4 According to Procedure 4, the ranks of a set of matrices given in (18) are required to be checked to find out if any of the eigenvalues of the matrix $A$ is a DOFM of the system $\mathcal{S}(\mathcal{K})$. It can be easily verified that the number of these matrices grows exponentially by $\nu_{\mu}$ (the number of complete bipartite subgraphs of $\left.\mathcal{G}_{\mu}\right)$. Therefore, in order to reduce the required computations, it is desirable to choose a graph $\mathcal{G}_{\mu}$ which has the minimum number of complete bipartite subgraphs among the set of graphs $\left\{\mathcal{G}_{1}, \ldots, \mathcal{G}_{l}\right\}$. If there is more than one such candidate, the one with fewer number of vertices is more preferable.

Corollary 2 The system $\mathcal{S}(\mathcal{K})$ is stabilizable by means of LTI decentralized overlapping controllers if and only if it does not have any DOFM in the closed right-half plane with respect to the information flow matrix $\mathcal{K}$.

Proof: The proof follows immediately from Theorem 4. 


\section{Numerical example}

Consider a system $\mathcal{S}$ with the following state-space matrices:

$$
\begin{aligned}
A & =\left[\begin{array}{cccccc}
6 & 2 & 2 & 4 & 2 & 5 \\
-9 & -5 & -3 & -4 & -3 & -6 \\
0 & 0 & -3 & 0 & 0 & 0 \\
-1 & -1 & -1 & -3 & -1 & -1 \\
9 & 6 & 3 & 4 & 4 & 6 \\
-8 & -2 & -2 & -4 & -2 & -7
\end{array}\right], \\
B & =\left[\begin{array}{cccc}
0 & -4 & 1 & 1 \\
0 & 7 & -1 & -4 \\
0 & 1 & 0 & 0 \\
0 & 1 & 0 & -1 \\
1 & -7 & 1 & 4 \\
0 & 7 & -1 & -1
\end{array}\right], \\
C & =\left[\begin{array}{cccccc}
2 & 0 & 1 & 0 & 0 & 1 \\
0 & 1 & 1 & 0 & 1 & 0 \\
1 & 0 & -4 & 0 & 0 & 1 \\
0 & -1 & -4 & 4 & 0 & 1
\end{array}\right], \quad D=\left[\begin{array}{llll}
0 & 0 & 1 & 2 \\
0 & 4 & 6 & 7 \\
0 & 0 & 1 & 0 \\
3 & 0 & 0 & 0
\end{array}\right]
\end{aligned}
$$

It can be easily verified that the modes of the system $\mathcal{S}$ are $-3,-3,-2,-2,1,1$. It is desired to design a stabilizing LTI controller for this system with the following control structure:

$$
\mathbf{K}=\left[\begin{array}{cccc}
0 & 0 & k_{13} & k_{14} \\
0 & k_{22} & k_{23} & k_{24} \\
0 & 0 & k_{33} & k_{34} \\
k_{41} & 0 & 0 & 0
\end{array}\right]
$$

Using Procedure 1, the graph $\mathcal{G}$ can be straightforwardly found, as depicted in Figure 3. Procedure 2 can now be utilized to obtain a number of specific graphs denoted by $\mathcal{G}_{1}, \mathcal{G}_{2}, \ldots, \mathcal{G}_{\mu}$ associated with the graph $\mathcal{G}$. For an arbitrary numbering of these graphs, let the graph $\mathcal{G}_{1}$ be the one drawn in Figure 4. The matrix $\mathbf{K}_{1}$ is attained from the graph $\mathcal{G}_{1}$ accordingly by means of Procedure 3 as follows:

$$
\mathbf{K}_{1}=\left[\begin{array}{cccccccc}
k_{13} & k_{14} & 0 & 0 & 0 & 0 & 0 & 0 \\
0 & 0 & k_{22} & k_{23} & k_{24} & 0 & 0 & 0 \\
0 & 0 & 0 & 0 & 0 & k_{33} & k_{34} & 0 \\
0 & 0 & 0 & 0 & 0 & 0 & 0 & k_{41}
\end{array}\right]
$$

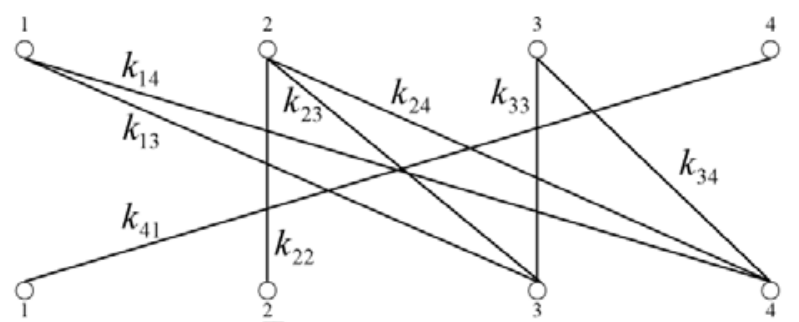

Fig. 3. The graph $\mathcal{G}$ corresponding to the system $\mathcal{S}$ given by $(19)$.

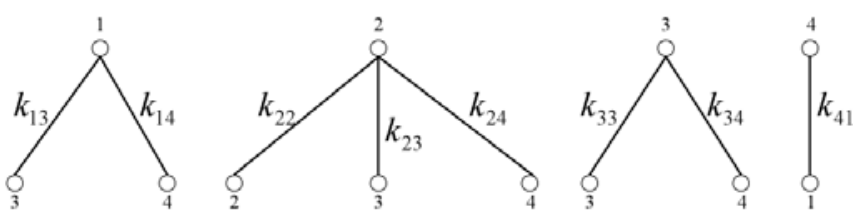

Fig. 4. The graph $\mathcal{G}_{1}$ obtained from $\mathcal{G}$ sketched in Figure 3.

It follows from Theorem 1 that there exist two binary matrices $\Phi_{1}$ and $\bar{\Phi}_{1}$ of proper dimensions such that $\mathbf{K}=$ $\Phi_{1} \mathbf{K}_{1} \bar{\Phi}_{1}$. These two matrices can be obtained from Theorem 2 as $\Phi_{1}=I$ and:

$$
\bar{\Phi}_{1}=\left[\begin{array}{llllllll}
0 & 0 & 0 & 0 & 0 & 0 & 0 & 1 \\
0 & 0 & 1 & 0 & 0 & 0 & 0 & 0 \\
1 & 0 & 0 & 1 & 0 & 1 & 0 & 0 \\
0 & 1 & 0 & 0 & 1 & 0 & 1 & 0
\end{array}\right]^{T}
$$

Consequently, the four-channel system $\mathcal{S}_{1}$ will have the following state-space representation:

$$
\begin{aligned}
& \dot{\mathbf{x}}_{1}(t)=A \mathbf{x}_{1}(t)+B \Phi_{1} \mathbf{u}_{1}(t) \\
& \mathbf{y}_{1}(t)=\bar{\Phi}_{1} C \mathbf{x}_{1}(t)+\bar{\Phi}_{1} D \Phi_{1} \mathbf{u}_{1}(t)
\end{aligned}
$$

Exploiting the method given in Davison and Chang (1990), one can easily conclude that the system $\mathcal{S}_{1}$ has an unstable DFM at $\lambda=1$ with respect to the control structure $\mathbf{K}_{1}$. Therefore, from the discussion given in the main results of the paper, the system $\mathcal{S}$ has an unstable DOFM at 1 with respect to the control interaction structure $\mathbf{K}$. Thus, there is no LTI structurally constrained controller complying with $\mathbf{K}$ to stabilize the system $\mathcal{S}$.

To arrive at the conclusion that the mode $\lambda=1$ is a DFM of the four-channel system $\mathcal{S}_{1}$ using the method proposed in Davison and Chang (1990), the rank of $2^{4}$ matrices of dimensions between 6 and 14 must be checked. In order to reduce this computational burden, it is preferable to attain a graph through Procedure 2 with the properties that the number of its vertices and also the number of its complete bipartite subgraphs are both relatively small (see Remark 4). For instance, instead of the graph $\mathcal{G}_{1}$ derived from $\mathcal{G}$, one can consider the graph $\mathcal{G}_{2}$ depicted 
in Figure 5, which is also found using Procedure 2. In this case, the matrix $\mathbf{K}_{2}$ can be obtained as:

$$
\mathbf{K}_{2}=\left[\begin{array}{cccc}
k_{13} & k_{14} & 0 & 0 \\
k_{23} & k_{24} & 0 & 0 \\
k_{33} & k_{34} & 0 & 0 \\
0 & 0 & k_{22} & 0 \\
0 & 0 & 0 & k_{41}
\end{array}\right]
$$

The three-channel system $\mathcal{S}_{2}$ can be attained in the same way that $\mathcal{S}_{1}$ was formed. To show that the mode $\lambda=1$ is a DFM of $\mathcal{S}_{2}$, the rank of $2^{3}$ matrices of dimensions between 6 and 11 must be checked. This demonstrates that the computation time can be noticeably diminished by commencing from the graph $\mathcal{G}_{2}$ rather than $\mathcal{G}_{1}$. This observation is an indication of the fact that the proper choice of graph in Procedure 2 can be quite crucial.
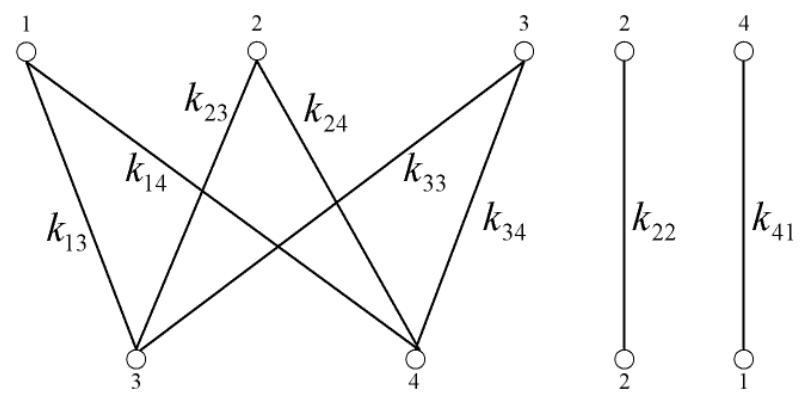

Fig. 5. The graph $\mathcal{G}_{2}$ obtained from $\mathcal{G}$ sketched in Figure 3.

\section{Conclusions}

This work tackles the control design problem for the systems with a constrained control structure. The notion of decentralized fixed mode (DFM) which has been introduced in the literature is extended here to define the new notion of decentralized overlapping fixed mode (DOFM). This definition is used to characterize those modes of a system which are fixed with respect to any LTI controller with a given overlapping structure. The primary goal of this work is to identify the DOFMs of the system efficiently. This is accomplished by employing a graph-theoretic approach. More precisely, a graph is obtained from which one can transform the overlapping structure of the controller into a decentralized structure for some other system. This brings about relating the DOFMs of the system to the DFMs of another system properly. Furthermore, a simple procedure is proposed to achieve pole-placement for the system by means of LTI structurally constrained controllers. The results obtained are illustrated in a numerical example.

\section{References}

Anderson, B. D. O. (1982). Transfer function matrix description of decentralized fixed modes. IEEE Transactions on Automatic Control. 27(6), 1176-1182.

Anderson, B. D. O., \& Clements, D. J. (1981). Algebraic characterizations of fixed modes in decentralized systems. Automatica. 17(5), 703-712.

Anderson, B. D. O., \& Moore, J. (1981). Timevarying feedback laws for decentralized control. IEEE Transactions on Automatic Control. 26(5), 1133-1139.

Bakule, L., Rodellar, J., \& Rossell, J. M. (2003). Contractibility of dynamic LTI controllers using complementary matrices. IEEE Transactions on Automatic Control. 48(7), 1269-1274.

Bakule, L., Rodellar, J., \& Rossell, J. M. (2001). Controllability-observability of expanded composite systems. Linear Algebra and its Applications. 332-334, 381-400.

Becerril, R., \& Aghdam, A. G. (2007). Decentralised nonlinear control with disturbance rejection for onramp metering in highways. IEE Proceedings, Control Theory and Applications. 1(1), 253-262.

Chu, D., \& Šiljak , D. D. (2005). A canonical form for the inclusion principle of dynamic systems. SIAM Journal on Control and Optimization. 44(3), 969990.

Davison, E. J., \& Chang, T. N. (1990). Decentralized stabilization and pole assignment for general proper systems. IEEE Transactions on Automatic Control. 35(6), 652-664.

Davison, E. J., \& Wang, S. H. (1985). A characterization of decentralized fixed modes in terms of transmission zeros. IEEE Transactions on Automatic Control. 30(1), 81-82.

Gong, Z., \& Aldeen, M. (1997). Stabilization of decentralized control systems. Journal of Mathematical Systems, Estimation, and Control. 7(1), 1-16.

Groumpos, P. P. (1994). Structural modelling and optimisation of large scale systems. IEE Control Theory and Applications. 141(1), 1-11.

Iftar, A. (1993). Overlapping decentralized dynamic optimal control. International Journal of Control. 58(1), 187-209.

Iftar, A. (1991). Decentralized optimal control with overlapping decompositions. IEEE International Conference on Systems Engineering (pp. 299-302).

Lafferriere, G., Caughman, J., \& Williams, A. (2004). Graph theoretic methods in the stability of vehicle formations. American Control Conference (pp. 3729-3734).

Lavaei, J., Momeni, A., \& Aghdam, A. G. (2007). Spacecraft formation control in deep space with reduced communication requirement. to appear in IEEE Transactions on Control Systems Technology.

Leros, A. P., \& Groumpos, P. P. (1987). The timeinvariant BAS decentralized large-scale linear reg- 
ulator problem. International Journal of Control. 46(1), 129-152.

Li, K., Kosmatopoulos, E. B., Ioannou, P., \& Boussalis, H. (1999). Centralized, decentralized, and overlapping control designs for a segmented telescope. in Proc. IEEE International Symposium on Intelligent Control/Intelligent Systems and Semiotics (pp. 173-178).

Li, H., \& Wang, Y. (2005). Nonlinear robust decentralized control of multimachine power systems. in Proc. 7th International Power Engineering Conference (pp. 1-6).

Mayeda, H. (1981). On structural controllability theorem. IEEE Transactions on Automatic Control. 26(3), 795-798.

Movsichoff, B. A., Lagoa, C. M., \& Che, H. (2005). Decentralized optimal traffic engineering in connectionless networks. IEEE Journal on Selected Areas in Communications. 23(2), 293-303.

Pichai, V., Sezer, M. E., \& Šiljak, D. D. (1984). A graph-theoretic characterization of structurally fixed modes. Automatica. 20(2), 247-250.

Ravi, M. S., Rosenthal, J., \& Wang, X. A. (1995). On decentralized dynamic pole placement and feedback stabilization. IEEE Transactions on Automatic Control. 40(9), 1603-1614.

Reinschke, K. (1988). Multivariable control: a graphtheoretic approach. Springer, Berlin.

Šiljak, D. D. (1991). Decentralized control of complex systems, Cambridge: Academic Press.

Šiljak, D. D., \& Zecevic, A. I. (2005). Control of largescale systems: Beyond decentralized feedback. Annual Reviews in Control. 29(2), 169-179.

Smith, R. S., \& Hadaegh, F. Y. (2002). Control topologies for deep space formation flying spacecraft. in Proc. 2002 American Control Conference (pp. 28362841).

Stankovic, S. S., Stanojevic, M. J., \& Šiljak, D. D. (2000). Decentralized overlapping control of a platoon of vehicles. IEEE Transactions on Control Systems Technology. 8(5), 816-832.

Wang, S. H., \& Davison, E. J. (1973). On the stabilization of decentralized control systems. IEEE Transactions on Automatic Control. 18(5), 473-478.

Zecevic, A. I., \& Šiljak, D. D. (2005). A new approach to control design with overlapping information structure constraints. Automatica. 41(2), 265-272. 